\title{
Bai-Hu-Tang, Ancient Chinese Medicine Formula, May Provide a New Complementary Treatment Option for Sepsis
}

\author{
Chien-Jung Lin, ${ }^{1,2}$ Yi-Chang Su, ${ }^{2,3}$ Cheng-Hung Lee, ${ }^{2}$ Tsai-Chung Li, \\ Yun-An Chen, ${ }^{3}$ and Sunny Jui-Shan Lin ${ }^{2,3}$ \\ ${ }^{1}$ Department of Chinese Medicine, National Defense Medical Center, Tri-Service General Hospital, Taipei 11490, Taiwan \\ ${ }^{2}$ Graduate Institute of Chinese Medicine, College of Chinese Medicine, China Medical University, No. 91 Hsueh-Shih Road, \\ Taichung 40402, Taiwan \\ ${ }^{3}$ School of Chinese Medicine, College of Chinese Medicine, China Medical University, Taichung 40402, Taiwan \\ ${ }^{4}$ Graduate Institute of Biostatistics, College of Public Health, China Medical University, Taichung 40402, Taiwan \\ ${ }^{5}$ Department of Healthcare Administration, College of Health Science, Asia University, Taichung 41354, Taiwan
}

Correspondence should be addressed to Sunny Jui-Shan Lin; taco423@ms26.hinet.net

Received 7 January 2013; Revised 19 April 2013; Accepted 25 April 2013

Academic Editor: Martin Kohlmeier

Copyright (C) 2013 Chien-Jung Lin et al. This is an open access article distributed under the Creative Commons Attribution License, which permits unrestricted use, distribution, and reproduction in any medium, provided the original work is properly cited.

Bai-Hu-Tang (BHT) has been broadly applied to treating the early stage of acute infection with systemic inflammation for two thousand years in Chinese medicine. We explore whether BHT is beneficial in treating sepsis and its effects on proinflammatory cytokine, interleukin-6, and anti-inflammatory cytokine interleukin-10, in which both play key roles in the progress of sepsis. Thirty-six male Sprague-Dawley rats were randomized into six groups, with cecal ligation and puncture (CLP) performed in all but the sham-control group. Rats in CLP $+\mathrm{BHT}^{-\mathrm{L}_{6}}$ and CLP $+\mathrm{BHT}^{-\mathrm{H}_{6}}$ groups, respectively, received a low $(0.45 \mathrm{~g} / \mathrm{kg})$ and high doses $(0.9 \mathrm{~g} / \mathrm{kg})$ of BHT, $6 \mathrm{hrs}$ postoperatively. CLP + BHT-L $\mathrm{L}_{12}$ and CLP + BHT- $\mathrm{H}_{12}$ groups, respectively, received low and high doses of BHT, 12 hrs postoperatively. Sham-control and sepsis-control groups received distilled water $(1 \mathrm{~mL})$ as vehicle, 6 hrs postoperatively. Serial blood samples were drawn before operation, as baseline, and at 4, 8, and $12 \mathrm{hrs}$ postoperatively for IL-6 and IL-10 assay. All rats were monitored for 3 days for survival study. Rats in the CLP + BHT- $\mathrm{H}_{6}$ group had significantly higher survival rate (80\%) and significantly lower levels of both IL-6 and IL-10 at 12 hrs postoperatively than those in the sepsis-control group. Results suggested that BHT may be a new complementary treatment option for sepsis.

\section{Introduction}

The term "translational research" first appeared in PubMed in 1993 , and it has cropped up in hundreds of articles each year after 2000. Relatively recent, a chasm has opened up between biomedical researchers and the patients who need their discoveries $[1,2]$. Enormous resources have been thrown into biomedical research, but the resulting commensurate gains are not as expected [1]. To bridge the gap between basic and clinical research, thriving treasures recorded in the Chinese medicine literature are worthy to be refined to provide promising targets since series of theoretical systems and practical experience of Chinese medicine were constructed through fighting diseases in the past thousands of years.
Sepsis is the leading cause of death in intensive care patients, with a reported incidence of more than 750,000 people each year in North America and Europe [3, 4]. Despite major advances in the understanding of its pathogenesis, no satisfactory therapy has emerged $[5,6]$; mortality from severe sepsis remains high at approximately $28 \%$ [3].

The most prominent pathological feature of sepsis is the exuberant, poorly regulated cytokine-mediated inflammatory response to microbial products [7]. Release of proinflammatory cytokines prolongs the inflammatory reaction [8] and leads to organ injury or dysfunction [9-12]. To prevent severe damage, anti-inflammatory cytokines are released. With overproduction of anti-inflammatory cytokines being occurred, the host becomes immunosuppressed, which can 
TABLE 1: Study groups and their interventions.

\begin{tabular}{|c|c|c|}
\hline Groups & Model & Administration (timing) \\
\hline Sham-control $(n=6)$ & Sham operation & Distilled water $1 \mathrm{~mL}$ as vehicle (6 hrs after operation) \\
\hline Sepsis-control $(n=6)$ & CLP & Distilled water $1 \mathrm{~mL}$ as vehicle (6 hrs after CLP) \\
\hline $\mathrm{CLP}+\mathrm{BHT}^{-\mathrm{L}_{6}}(0.45 \mathrm{~g} / \mathrm{kg})(n=6)$ & CLP & BHT $0.5 \mathrm{~mL}$ + distilled $0.5 \mathrm{~mL}$ (6 hrs after CLP) \\
\hline $\mathrm{CLP}+\mathrm{BHT}^{-\mathrm{H}_{6}}(0.9 \mathrm{~g} / \mathrm{kg})(n=6)$ & CLP & BHT $1 \mathrm{~mL}(6 \mathrm{hrs}$ after CLP) \\
\hline $\mathrm{CLP}+\mathrm{BHT}^{-\mathrm{L}_{12}}(0.45 \mathrm{~g} / \mathrm{kg})(n=6)$ & CLP & BHT $0.5 \mathrm{~mL}+$ distilled $0.5 \mathrm{~mL}$ (12 hrs after CLP) \\
\hline $\mathrm{CLP}+\mathrm{BHT}^{-\mathrm{H}_{12}}(0.9 \mathrm{~g} / \mathrm{kg})(n=6)$ & CLP & BHT $1 \mathrm{~mL}(12 \mathrm{hrs}$ after CLP) \\
\hline
\end{tabular}

CLP: rats received cecal ligation and puncture.

BHT- $\mathrm{L}_{6}$ : low dose of BHT was orally administered $6 \mathrm{hrs}$ postoperatively.

BHT- $\mathrm{H}_{6}$ : high dose of BHT was orally administered 6 hrs postoperatively.

BHT- $\mathrm{L}_{12}$ : low dose of BHT was orally administered $12 \mathrm{hrs}$ postoperatively.

BHT- $\mathrm{H}_{12}$ : high dose of BHT was orally administered $12 \mathrm{hrs}$ postoperatively.

lead to death $[13,14]$. A recent large observational study of sepsis revealed mortality highest when proinflammatory and anti-inflammatory cytokine levels are high [15]. These findings demonstrate that limiting both proinflammatory and anti-inflammatory cytokines should be considered in treating sepsis.

Bai-Hu-Tang (BHT, also known as White Tiger Decoction), a widely used Chinese medicine formula composed of Shi-Gao (Gypsum Fibrosum), Zhi-Mu (Rhizoma Anemarrhenae), Zhi-Gan-Cao (Radix Glycyrrhizae Preparata), and Geng-Mi (nonglutinous rice), has been extensively used in the early stage of acute infection with systemic inflammation for two thousand years in Chinese medicine [16-18], yet no empirical studies conducted. We explored treatment effects of BHT against cecal ligation and puncture- (CLP-) induced sepsis in rats. Since plasma levels of interleukin-6 (IL-6) and interleukin-10 (IL-10) were demonstrated as good parameters to predict the outcome of sepsis in our previous study [19], survival rate and plasma levels of IL- 6 and IL-10 of rats were measured in the present study. Furthermore, mean arterial pressure (MAP) and heart rate (HR) were also measured to monitor the development and progression of sepsis.

\section{Materials and Methods}

2.1. Drugs and Preparation. Bai-Hu-Tang concentrated extract powder is composed of four ingredients: Shi-Gao (Gypsum Fibrosum), Zhi-Mu (Rhizoma Anemarrhenae), Zhi-GanCao (Radix Glycyrrhizae Preparata), and Geng-Mi (nonglutinous rice) with the proportion of $3: 8: 1: 4$. We purchased the formula from a renowned GMP manufacturer of concentrated herbal extracts which conforms to international standards (Sun-Ten Pharmaceutical CO., Taipei; its products can be purchased in Asia, Europe, and North America.). When in use, BHT was prepared by mixing 1 gram of extract powder with $5 \mathrm{~mL}$ of warm sterile distilled water $\left(30^{\circ} \mathrm{C}\right)$.

2.2. Animal Model. A total of 36 male Sprague-Dawley (SD) rats, weighing 250 to $300 \mathrm{~g}$, were obtained from BioLASCO Taiwan Co. Ltd. and then maintained at a constant $25^{\circ} \mathrm{C}$ with free access to pelleted food and water in a room with $12 \mathrm{~h}$ light/dark cycle. Sepsis was induced by CLP as previously described $[19,20]$. Briefly, rats were anesthetized with ether, with blood samples obtained from the tail artery. After that, a $2 \mathrm{~cm}$ midline incision was made, and then the cecum was exteriorized. Ligation was performed below the ileocecal valve without causing intestinal obstruction. Ligated cecum was punctured twice with a 20 -gauge needle at the antimesenteric surface and then gently squeezed to extrude adequate amount of stool through two puncture wounds. The bowel was then replaced in the peritoneal cavity and abdomen closed in layers with 3-O silk sutures. Finally, warm saline $\left(3 \mathrm{~mL} / 100 \mathrm{~g}\right.$ body weight, $37^{\circ} \mathrm{C}$ ) was subcutaneously injected [20]. Sham-operated rats underwent the same treatment, except for ligation and puncture procedures.

The right carotid artery was then cannulated by PE-50 tubing (Clay Adams, Parsippany, NJ, USA) equipped with a heparin lock filled with heparin saline to permit blood pressure measurement, drawing of blood samples, and infusion of fluids. This catheter was passed through the subcutaneous tunnel to the back of the neck. Finally, rats were placed in cages with warm light and allowed to recover for half an hour; serial MAP and HR were measured and recorded by MP100 System (Biopac Inc., Santa Barbara, CA, USA) at 1, 4,8 , and $12 \mathrm{hrs}$ postoperatively by inserting the probe into the catheter via the heparin lock. Experimental protocols were approved by the Animal Experiment Committee of China Medical University and conducted according to the American Physiological Society guiding principles for the care and use of laboratory animals.

2.3. Study Groups. Thirty-six rats were randomly divided into six experimental groups of six rats each. According to prior study, after being subjected to CLP, the hyperdynamic state developed within $10 \mathrm{hrs}$; rats became obviously ill looking $12 \mathrm{hrs}$ postoperatively [20]. Timing of early oral administration of BHT was thus set at $6 \mathrm{hrs}$ postoperatively within the progress of the hyperdynamic state in our study because BHT is applied clinically when the following symptoms developed in the early stage of infectious diseases: high fever, presence of sweating, thirst, and flooding big pulse [17]. The time points we defined as early and late interventions at $6 \mathrm{hrs}$ and $12 \mathrm{hrs}$ postoperatively, respectively, were consistent with current international opinions $[21,22]$. This design was also aimed to compare the effects on survival between "early" and "late" administration of BHT. Table 1 details the intervention established in each group. 

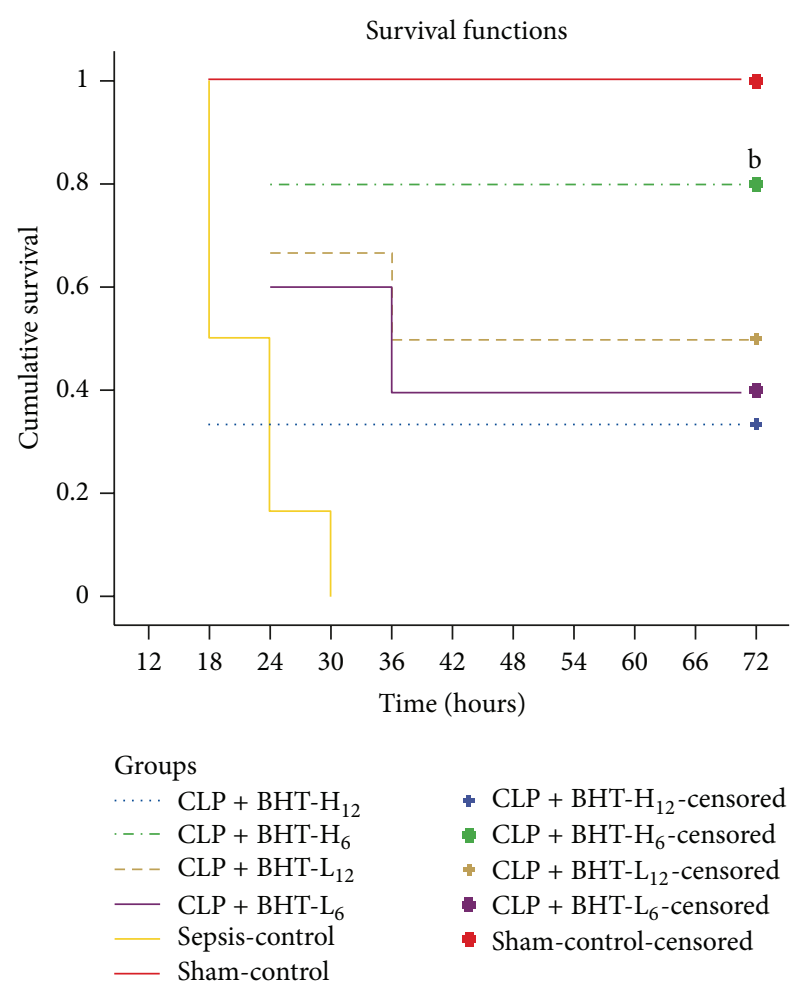

FIGURE 1: Kaplan-Meier survival curves of all groups. Sham-control group received sham operation and distilled water; the remaining groups received cecum ligation and puncture (CLP). All CLP rats received Bai-Hu-Tang (BHT), except for those in the sepsis-control group, which received distilled water. CLP $+\mathrm{BHT}^{-\mathrm{L}_{6}}$ : low dose of BHT $(0.45 \mathrm{~g} / \mathrm{kg})$ administered $6 \mathrm{hrs}$ postoperatively. CLP + BHT$\mathrm{H}_{6}$ : high dose of BHT $(0.9 \mathrm{~g} / \mathrm{kg})$ administered $6 \mathrm{hrs}$ postoperatively. CLP + BHT-L $_{12}$ : low dose BHT $(0.45 \mathrm{~g} / \mathrm{kg})$ administered $12 \mathrm{hrs}$ postoperatively. CLP $+\mathrm{BHT}_{12}$ : high-dose of BHT $(0.9 \mathrm{~g} / \mathrm{kg})$ administered $12 \mathrm{hrs}$ postoperatively. All rats were monitored for three days postoperatively for survival study. Notice that the survival was highest in the CLP $+\mathrm{BHT}^{-\mathrm{H}_{6}}$ group. ${ }^{\mathrm{b}} \mathrm{P}<0.05$, compared to sepsis-control group.

2.4. Survival Studies. All rats were monitored for three days postoperatively, those surviving for more than three days categorized in the survival group and the remainder in the nonsurvival group.

2.5. Collection of Serum Samples and Cytokine Assay. Before CLP and catheterization, a $0.5 \mathrm{~mL}$ blood sample was drawn from the tail artery to establish baseline value measurement of cytokines. After CLP and catheterization, $0.5 \mathrm{~mL}$ blood samples were taken from the heparin lock of the carotid artery catheter 4,8 , and $12 \mathrm{hrs}$ later. Normal saline $\left(0.5 \mathrm{~mL}, 37^{\circ} \mathrm{C}\right)$ was injected into the artery to replace blood volume each time. The blood samples were placed in $1.5 \mathrm{~mL}$ microtubes and centrifuged at $9000 \mathrm{rpm}$ for $5 \mathrm{~min}$ at $25^{\circ} \mathrm{C}$ to separate plasma. Serum samples were then removed and stored at $-80^{\circ} \mathrm{C}$ for further cytokine assay. Serum levels of IL- 6 and IL-10 were measured by the enzyme-linked immunosorbent assay (ELISA) via a commercially available kit ( $\&$ \& systems, MN55413, Minneapolis, MN, USA) according to manufacturer's specifications.
2.6. Statistical Analysis. Log-rank test with Bonferroni adjustment for multiple comparisons analyzed differences in survival among all groups.

Comparisons of MAP, HR, and plasma levels of IL-6 and IL-10 were analyzed only among the sham-control, sepsiscontrol, $\mathrm{CLP}+\mathrm{BHT}_{-} \mathrm{L}_{6}$, and CLP $+\mathrm{BHT}^{-\mathrm{H}_{6}}$ groups since we did not measure these four observation parameters in the CLP $+\mathrm{BHT}^{-\mathrm{L}_{12}}$ and $\mathrm{CLP}+\mathrm{BHT}^{-\mathrm{H}_{12}}$ groups. Because MAP, HR, and plasma levels of IL-6 and IL-10 were not normally distributed, Kruskal-Wallis was used for overall comparison and then Mann-Whitney $U$ with Bonferroni adjustment for multiple comparisons was adopted to detect the differences of these parameters at baseline, and at 4, 8, and $12 \mathrm{hrs}$ postoperatively among groups.

To examine the dose effect of BHT on survival and cytokine changes, we first derived an ordinal variable by coding the sepsis-control group as " 0 ," early administration of low-dose BHT (CLP + BHT- $\left.\mathrm{L}_{6}\right)$ as "0.5," and early administration of high-dose BHT (CLP + BHT-H $\left._{6}\right)$ as "1." Second, we defined changes of IL- 6 and IL-10 by subtracting plasma levels of cytokines at baseline from those at $12 \mathrm{hrs}$ postoperatively. All cytokine change values were natural log transformed. Then we applied simple linear regression to correlate doses of BHT with changes of IL- 6 and IL-10. Finally, we adopted Cox proportional hazards models to explore how doses of BHT correlated with survival through modulating cytokines. Statistical analyses used SPSS version 14.0 for Windows (SPSS, Chicago, IL, USA); $P$ value $<0.05$ was considered statistically significant.

\section{Results}

Totally, 36 rats were observed. However, two were excluded due to catheter obstruction. Therefore, the study population comprised 34 rats.

3.1. Effects of BHT on Survival. No rats died until $18 \mathrm{hrs}$ postoperatively. Overall test for comparing survival functions of each group was significant $(P<0.05)$. Kaplan-Meier survival curves are shown in Figure 1. The sham-control group had a $100 \%$ survival rate, the sepsis-control group $0 \%$ in survival. Survival rates were $40 \%$ for CLP $+\mathrm{BHT}_{-} \mathrm{L}_{6}, 80 \%$ for $\mathrm{CLP}+\mathrm{BHT}_{-} \mathrm{H}_{6}, 50 \%$ for CLP $+\mathrm{BHT}_{12}$, and $33 \%$ for $\mathrm{CLP}+\mathrm{BHT}_{-\mathrm{H}_{12}}$. Survival rate was significantly higher in the $\mathrm{CLP}+\mathrm{BHT}_{-} \mathrm{H}_{6}$ group (80\%) than in the sepsis-control group $(P<0.05)$.

3.2. Effects of BHT on Plasma Levels of IL-6 and IL-10. Figure 2 illustrates changes in plasma levels of IL-6 and IL10 , that is, similar to all groups at baseline and both increasing significantly after CLP in the sepsis-control, CLP + $\mathrm{BHT}_{-} \mathrm{L}_{6}$, and CLP $+\mathrm{BHT}^{-\mathrm{H}_{6}}$ groups as compared with sham-control group $\left({ }^{\mathrm{a}} \mathrm{P}<0.05\right)$.

Early administration of high-dose BHT (CLP + BHT$\mathrm{H}_{6}$ ) significantly reduced elevations of IL-6 and IL-10 as compared with the sepsis-control group at $12 \mathrm{hrs}$ postoperatively ( ${ }^{\mathrm{b}} \mathrm{P}<0.05$, IL-6: 868.24 versus $3177.64 \mathrm{pg} / \mathrm{mL}$; IL10: 85.35 versus $229.45 \mathrm{pg} / \mathrm{mL}$ ). Likewise, compared with lowdose BHT administration group, rats in the high-dose group 

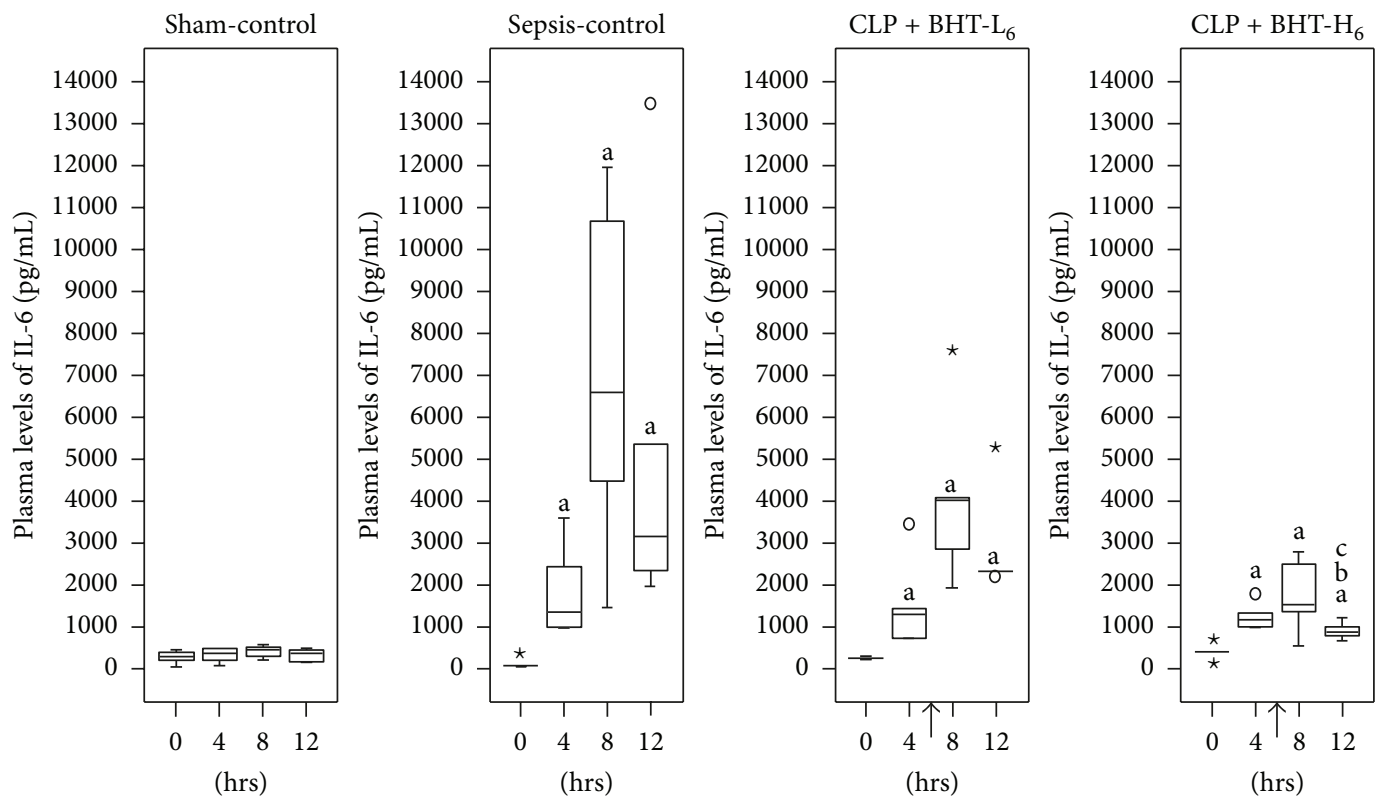

(a)
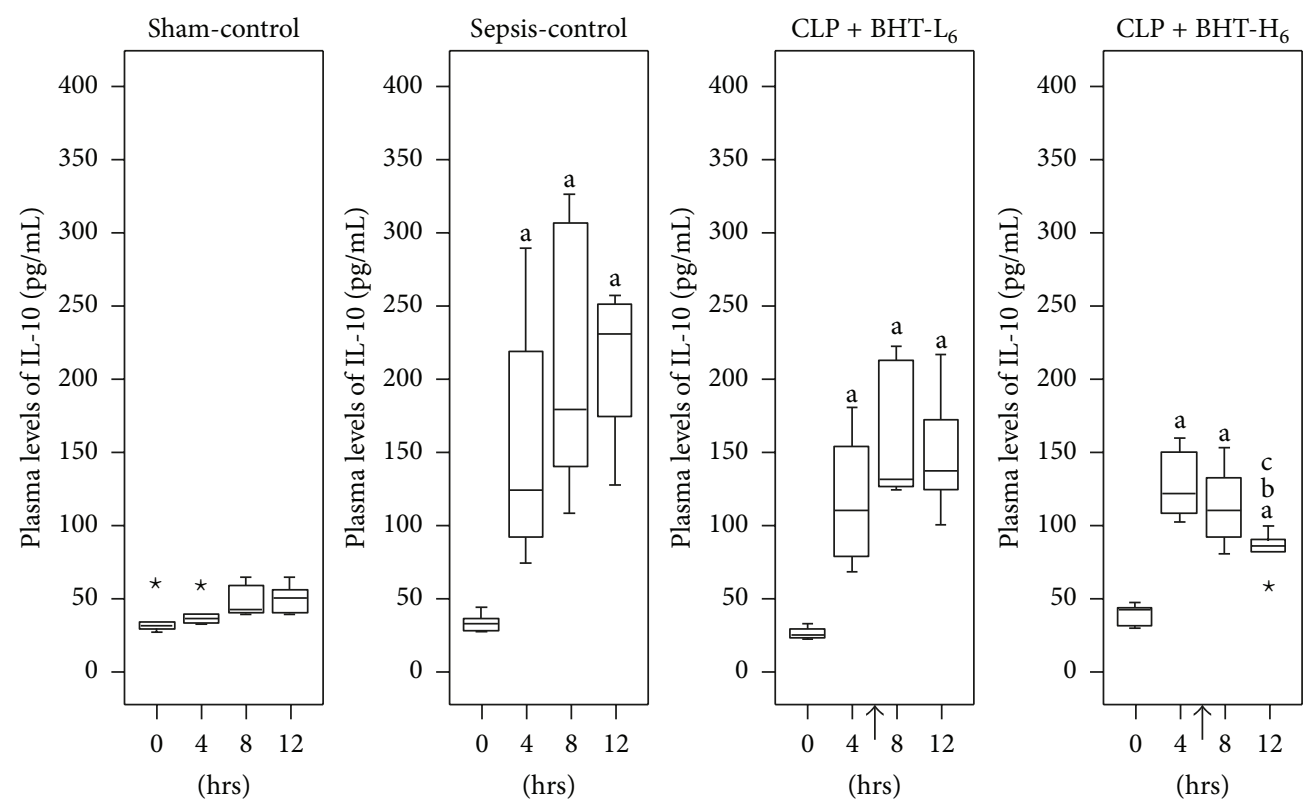

(b)

FIGURE 2: Effects of Bai-Hu-Tang on plasma levels of IL-6 (a) and IL-10 (b). Plasma levels of IL-6 and IL-10 in the sham-control, sepsis-control, $\mathrm{CLP}+\mathrm{BHT}_{-} \mathrm{L}_{6}$, and CLP + BHT- $\mathrm{H}_{6}$ groups at $0,4,8$, and 12 hours postoperatively were shown above. Boxes denote 25 th and 75 th percentile;

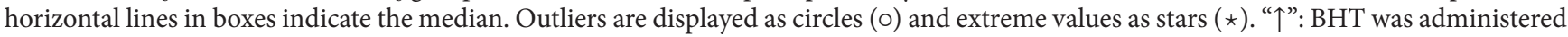
at 6 hrs postoperatively. Notice that the elevations of IL- 6 and IL-10 levels were both reduced after the administration of BHT and it was most prominent in the CLP + BHT- $\mathrm{H}_{6}$ group. ${ }^{\mathrm{a}} \mathrm{P}<0.05$, compared with sham-control group. ${ }^{\mathrm{b}} \mathrm{P}<0.05$, compared with sepsis-control group. ${ }^{\mathrm{c}} \mathrm{P}<0.05$, compared with $\mathrm{CLP}+\mathrm{BHT}^{-\mathrm{L}_{6}}$ group.

had significantly lower plasma levels of IL-6 and IL-10 at 12 hrs postoperatively $\left({ }^{c} P<0.05\right.$, IL-6 median: 868.24 versus $2346.94 \mathrm{pg} / \mathrm{mL}$, IL-10 median: 85.35 versus $136.32 \mathrm{pg} / \mathrm{mL}$ ).

3.3. Effect of BHT on MAP and HR. No significant difference in MAP arose among four groups at baseline and postoperatively (Figure 3). HR were similar in all groups at baseline and increased significantly after CLP, as compared with the sham-control group $\left({ }^{\mathrm{a}} P<0.05\right)$. No significant difference appeared for any BHT treatment group versus sepsis-control group (Figure 4).

3.4. Association among BHT, Cytokine Changes, and Survival Rate. Table 2 plots linear regression model for association 

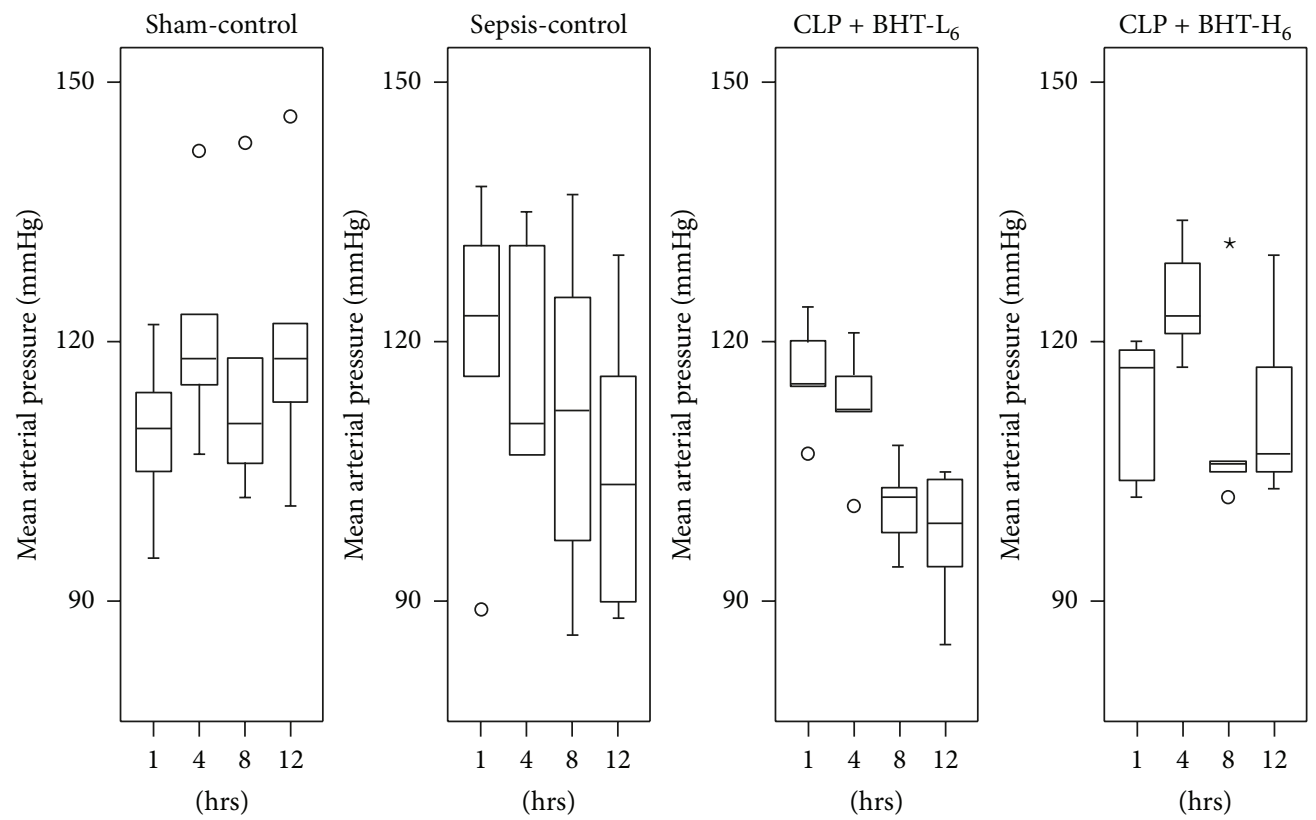

Figure 3: Effects of Bai-Hu-Tang on mean arterial pressure (MAP). MAP in sham-control, sepsis-control, CLP + BHT- $\mathrm{L}_{6}$, and CLP + BHT- $\mathrm{H}_{6}$ groups at $0,4,8$, and 12 hours after operation were expressed as the box plots. Boxes denote the 25th and 75th percentile; horizontal lines in the box indicate the median. Outliers are displayed as circles (o) and extreme values as stars $(\star)$. No significant difference was noted at any time point among the four groups.

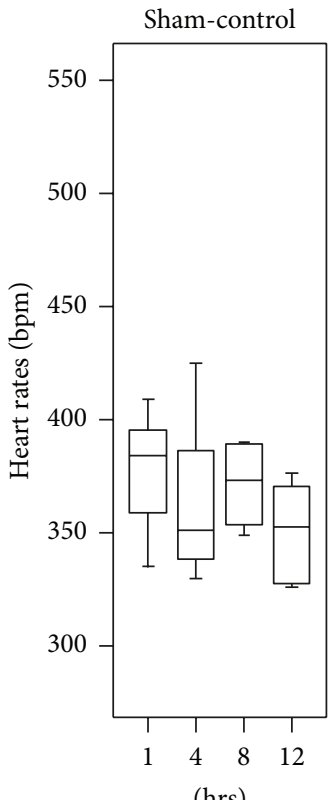

(hrs)
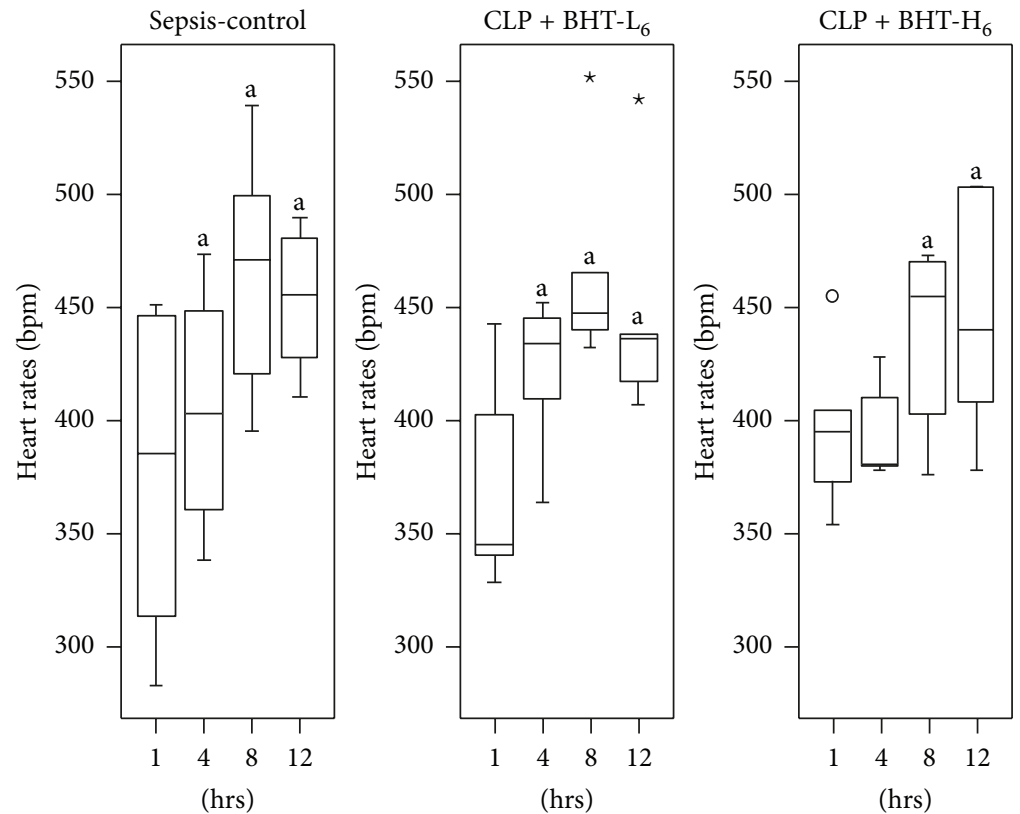

FIGURE 4: Effects of Bai-Hu-Tang on heart rates (HR). HR in the sham-control, sepsis-control, CLP $+\mathrm{BHT}-\mathrm{L}_{6}$, and CLP $+\mathrm{BHT} \mathrm{H}_{6}$ groups at $0,4,8$, and 12 hours after operation were shown above. Boxes denote 25 th and 75 th percentile; horizontal lines in the box indicate the median. Outliers are displayed as circles $(\circ)$ and extreme values as stars $(\star)$. Notice that HR elevated in all groups after CLP. ${ }^{\mathrm{a}} P<0.05$, compared with sham-control group.

between different doses of BHT and cytokine changes. We found significant negative correlation between a high dose of BHT and change in IL- 6 and IL-10 $(\beta=-2.13$ and -1.41 , resp., both $P<0.001$ ). The model thus proved effects of BHT on changes of IL- 6 and IL-10 as inverse and dose-dependent $(\beta=-2.09,-1.39, P$ for trend $<0.001)$. Table 3 displays results of Cox regression models. Before adjustment, high dose BHT manifested negative correlation with risk of death (hazard ratio: 0.08; 95\% confidence interval (CI): 0.01-0.73) $(P<0.05)$; changes in IL-6 and IL-10 both exhibited 
TABLE 2: Linear regression model of association between different doses of BHT and cytokine changes.

\begin{tabular}{|c|c|c|c|c|c|c|}
\hline & \multicolumn{3}{|c|}{ Change of IL- $6^{\mathrm{b}}$} & \multicolumn{3}{|c|}{ Change of IL-10 ${ }^{b}$} \\
\hline & $\beta$ & $P$ value & $P$ for trend & $\beta$ & $P$ value & $P$ for trend \\
\hline Dose of BHT & -2.09 & & $<0.001^{*}$ & -1.39 & & $<0.001^{*}$ \\
\hline High dose $(1)^{\mathrm{a}}$ & -2.13 & $<0.001^{*}$ & & -1.41 & $<0.001^{*}$ & \\
\hline Low dose $(0.5)^{\mathrm{a}}$ & -0.39 & 0.295 & & -0.38 & 0.081 & \\
\hline
\end{tabular}

TABLE 3: Cox proportional hazards models of survival rates for different doses of BHT and changes of IL-6 and IL-10.

\begin{tabular}{|c|c|c|c|c|}
\hline \multirow{2}{*}{ Covariate } & \multicolumn{2}{|c|}{ Unadjusted } & \multicolumn{2}{|c|}{ Adjusted $^{+}$} \\
\hline & $\mathrm{HR}(95 \% \mathrm{CI})$ & $P$ value & HR $(95 \%$ CI $)$ & $P$ value \\
\hline \multicolumn{5}{|l|}{ Dose of BHT } \\
\hline High dose $(1)^{\mathrm{a}}$ & $0.08(0.01-0.73)$ & $0.025^{*}$ & $0.56(0.02-20.17)$ & 0.751 \\
\hline Low dose $(0.5)^{\mathrm{a}}$ & $0.26(0.06-1.19)$ & 0.083 & $0.25(0.05-1.40)$ & 0.115 \\
\hline Sepsis control $(0)^{\mathrm{a}}$ & & & Reference & \\
\hline \multicolumn{5}{|l|}{ Cytokine changes } \\
\hline Change of IL- $6^{\mathrm{b}}$ & $3.58(1.50-8.56)$ & $0.004^{*}$ & $3.87(1.03-14.53)$ & $0.045^{*}$ \\
\hline Change of IL- $10^{\mathrm{b}}$ & $4.56(1.50-13.84)$ & $0.007^{*}$ & $0.82(0.10-6.91)$ & 0.851 \\
\hline
\end{tabular}

ardinal variable: coding the early administration of high-dose BHT (CLP + BHT- $\left.\mathrm{H}_{6}\right)$ as "1," and the early administration of low-dose BHT administration $\left(\mathrm{CLP}+\mathrm{BHT}^{-\mathrm{L}_{6}}\right)$ as “0.5”.

${ }^{\mathrm{b}}$ Changes of IL-6 and IL-10: subtracting the plasma levels of cytokines at baseline from those at 12 hrs postoperatively.

All values were natural log transformed.

Unadjusted: univariate Cox proportional hazards model.

Adjusted $^{+}$: for different doses of BHT, changes of IL-6 and IL-10.

HR: hazard ratio, CI: confidence interval, ${ }^{*} P<0.05$.

significant positive correlation with individual risk of death (hazard ratio: 3.58 and 4.56 ; $95 \%$ (CI): $1.50-8.56$ and $1.50-$ 13.84) $(P<0.05)$. Accounting for diverse BHT doses and cytokine changes, IL-6 variation was the sole predictor for survival, estimated at nearly fourfold greater risk of death (adjusted hazard ratio: 3.87; 95\% (CI): 1.03-14.53) $(P<0.05)$.

\section{Discussion}

To our knowledge, this is the first study to explore treatment effects of BHT on survival and cytokine regulation in CLPinduced septic rats. Our results show early administration of high-dose BHT significantly improving survival by reducing IL-6 level. Although IL-10 was simultaneously reduced, modulating effects of BHT on IL-10 did not have significant effects on survival. In addition, we revealed effects of BHT on cytokine changes as dose dependent.

The study design of this study is based on our previous report [19], in which the prognosis of septic rats was followed up for 3 days after CLP, and IL- 6 and IL-10 were found to be good predictors of sepsis mortality. Therefore, this time we adopted the same experiment protocol and administered BHT only one time after sepsis induction, in order to clearly evaluate the effects of BHT on both cytokines changes and survival rate. The protocol to monitor the septic rats for the first 3 days after sepsis induction is the same as the method reported in similar studies of sepsis $[23,24]$. Besides, once the septic rats can survive more than 3 days, most of them will survive thereafter.

There has been no satisfying improvement in finding new therapies to decrease mortality of sepsis in the past 20 years. Many clinical trials and experiments suffered from design flows [25], for example, key biological information not accessed before initiating trial of an experimental agent [25]. Target biomarker should be present at the time of study entry. Serial measurement should better evaluate the immune status of sepsis [25]. Hence, our study measured baseline levels of cytokines before operation and obtained time serial plasma cytokines levels later, thus confirming IL-6 and IL-10 plasma levels as elevated already before BHT administration; their change patterns followed treatment intervention. These data lent insight into dynamic BHT immune-modulating effects. Simultaneously, time serial MAP and HR were measured, enabling us to gauge development and progression of sepsis in CLP rats.

Recently, both IL-6 and IL-10 elevations were found to be significantly correlated with mortality of sepsis $[15,26]$. Peng et al. revealed hemoadsorption decreasing risk of death from sepsis by removal of both IL-6 and IL-10 [27]. Our results concurred with these findings, yet there were several key features in our study. First, oral BHT administration was not as invasive as hemoadsorption or hemofiltration. Second, unlike hemoadsorption or hemofiltration applied in treating late-stage sepsis, appropriate treatment timing 
of BHT is at early stages of sepsis. Third, while proinflammatory cytokines, including IL-1, tumor necrosis factor alpha (TNF- $\alpha$ ), and IL- 6 , have been shown to induce cardiac myocyte apoptosis and necrosis and may cause damage to other organs, expression of inflammatory cytokines during specific response is not entirely deleterious. Inflammatory cytokines are necessary for limiting and eliminating local infections and increasing host survival [28, 29], for example, IL-6 required for successful outcome in sepsis, given its ability to promote hepatocyte recovery and regeneration [30]. These findings point to appropriate downregulation, albeit not extreme suppression, of proinflammatory cytokines as beneficial in treating sepsis. Our study results showed that the BHT administration decreases elevation of IL- 6 after CLP without totally blocking the expression of IL-6, which might not make the host suffer from immunosuppression.

On the other hand, magnitude of IL-10 response appears to correlate with both severity of inflammatory insult and plasma concentration of proinflammatory cytokines, such as TNF- $\alpha$ [31]. We found that IL-10 simultaneously reduced following early administration of high-dose BHT, but with no direct cause and effect between BHT administration and level of IL-10. These findings imply reduction of proinflammatory cytokine in early-stage sepsis as sequentially downregulating anti-inflammatory cytokine, IL-10. Hence, the mechanism by which BHT modulated IL-6 and whether BHT can modulate TNF- $\alpha$ or other proinflammatory cytokines should be evaluated in the future.

This study had several limitations. First, due to limited amount of blood volume we could draw from rats each time, we only observed plasma level of proinflammatory cytokine IL-6 and anti-inflammatory cytokine IL-10, with the main focus chosen from the literature review and based on results of our prior study $[15,19,26,32]$. Second, to probe treatment effects of BHT, our intervention only included BHT administration with limited fluid supplement (Normal Saline, $3 \mathrm{~mL} / 100 \mathrm{~g}$ body weight). Thus, we could not evaluate treatment effects of BHT applied in combination with current clinical therapies like antibiotics, fluid resuscitation, or inotropics. Third, before formally performing the experiment described in this manuscript, pilot studies were conducted to confirm the optimal dose of BHT adopted. The results of these pilot studies revealed the same trend that the group of septic rats which received BHT had higher survival rate in a dosedependent manner. Due to the limitation of grant resources, the sample size of each group in this experiment only received the average number of animal experiments [23, 24]. The relative small sample size is a limitation of our study, but the dose-dependent effects of BHT on both the survival rate and circulating IL- 6 and IL-10 still provide evidence of BHT as a new treatment option for sepsis.

\section{Conclusions}

For centuries, based on theories of traditional Chinese Medicine, BHT has been considered effective in treating early stage severe infection with systemic inflammation. Results of our study afforded scientific evidence for BHT in treating sepsis by a CLP model and its effects on cytokine modulation: early administration of high-dose BHT markedly improved survival by directly reducing plasma level of IL-6, and simultaneously decreased IL-10 was observed. Our results suggest BHT as a novel complementary therapeutic candidate in treating early sepsis besides antibiotic, fluid resuscitation, and inotropic therapy, though combined effects of BHT with these treatments still need further evaluation.

\section{Conflict of Interests}

All authors declare that there is no conflict of interests.

\section{Acknowledgments}

The authors would like to thank the Committee on Chinese Medicine and Pharmacy, Department of Health, Executive Yuan, Taiwan, for the Grants support (nos. CCMP95-RD-214 and CCMP96-RD-209).

\section{References}

[1] D. Butler, "Translational research: crossing the valley of death," Nature, vol. 453, no. 7197, pp. 840-842, 2008.

[2] S. H. Woolf, "The meaning of translational research and why it matters," Journal of the American Medical Association, vol. 299, no. 2, pp. 211-213, 2008.

[3] D. C. Angus, W. T. Linde-Zwirble, J. Lidicker, G. Clermont, J. Carcillo, and M. R. Pinsky, "Epidemiology of severe sepsis in the United States: analysis of incidence, outcome, and associated costs of care," Critical Care Medicine, vol. 29, no. 7, pp. 1303-1310, 2001.

[4] J. L. Vincent, Y. Sakr, C. L. Sprung et al., "Sepsis in European intensive care units: results of the SOAP study," Critical Care Medicine, vol. 34, no. 2, pp. 344-353, 2006.

[5] J. A. Russell, "Management of sepsis," The New England Journal of Medicine, vol. 355, no. 16, pp. 1699-1713, 2006.

[6] J. Carlet, "Prescribing indications based on successful clinical trials in sepsis: a difficult exercise," Critical Care Medicine, vol. 34, no. 2, pp. 525-529, 2006.

[7] M. P. Glauser, "Pathophysiologic basis of sepsis: considerations for future strategies of intervention," Critical Care Medicine, vol. 28, no. 9, pp. S4-S8, 2000.

[8] L. Liaudet, J. G. Mabley, F. G. Soriano et al., "Inosine reduces systemic inflammation and improves survival in septic shock induced by cecal ligation and puncture," American Journal of Respiratory and Critical Care Medicine, vol. 164, no. 7, pp. 12131220, 2001.

[9] M. W. N. Nijsten, C. E. Hack, M. Helle, H. J. ten Duis, H. J. Klasen, and L. A. Aarden, "Interleukin-6 and its relation to the humoral immune response and clinical parameters in burned patients," Surgery, vol. 109, no. 6, pp. 761-767, 1991.

[10] O. Murch, M. Abdelrahman, M. Collino et al., "Sphingosylphosphorylcholine reduces the organ injury/dysfunction and inflammation caused by endotoxemia in the rat," Critical Care Medicine, vol. 36, no. 2, pp. 550-559, 2008.

[11] G. van den Berghe, P. Wouters, F. Weekers et al., "Intensive insulin therapy in critically ill patients," The New England Journal of Medicine, vol. 345, no. 19, pp. 1359-1367, 2001.

[12] F. L. Yeh, H. D. Shen, and R. H. Fang, "Deficient transforming growth factor $\beta$ and interleukin-10 responses contribute to the 
septic death of burned patients," Burns, vol. 28, no. 7, pp. 631637, 2002.

[13] R. C. Bone, "Sir Isaac Newton, sepsis, SIRS, and CARS," Critical Care Medicine, vol. 24, no. 7, pp. 1125-1128, 1996.

[14] R. C. Bone, C. J. Grodzin, and R. A. Balk, "Sepsis: a new hypothesis for pathogenesis of the disease process," Chest, vol. 112, no. 1, pp. 235-243, 1997.

[15] J. A. Kellum, L. Kong, M. P. Fink et al., "Understanding the inflammatory cytokine response in pneumonia and sepsis: results of the Genetic and Inflammatory Markers of Sepsis (GenIMS) Study," Archives of Internal Medicine, vol. 167, no. 15, pp. 1655-1663, 2007.

[16] X. C. Xu, Pharmacology of Traditional Chinese Medical Formulae, Higher Educational Press, Beijing, China, 1994.

[17] C. Y. Liu, A. Tseng, and Y. Sue, Chinese Herbal Medicine: Modern Applications of Traditional Formulas, CRC Press, New York, NY, USA, 2005.

[18] G. Maciocia, The Foundations of Chinese Medicine: A Comprehensive Text for Acupuncturists and Herbalists, Elsevier Churchill Livingstone, Edinburgh, UK, 2005.

[19] C. H. Wang, M. J. Gee, C. Yang, and Y. C. Su, "A new model for outcome prediction in intra-abdominal sepsis by the linear discriminant function analysis of IL-6 and IL-10 at different heart rates," Journal of Surgical Research, vol. 132, no. 1, pp. 4651, 2006.

[20] K. A. Wichterman, A. E. Baue, and I. H. Chaudry, "Sepsis and septic shock: a review of laboratory models and a proposal," Journal of Surgical Research, vol. 29, no. 2, pp. 189-201, 1980.

[21] R. P. Dellinger, M. M. Levy, J. M. Carlet et al., "Surviving Sepsis Campaign: international guidelines for management of severe sepsis and septic shock: 2008," Critical Care Medicine, vol. 36, no. 1, pp. 296-327, 2008.

[22] D. Vyas, P. Javadi, P. J. Dipasco, T. G. Buchman, R. S. Hotchkiss, and C. M. Coopersmith, "Early antibiotic administration but not antibody therapy directed against IL-6 improves survival in septic mice predicted to die on basis of high IL-6 levels," American Journal of Physiology, vol.289, no. 4, pp. R1048-R1053, 2005.

[23] K. P. Rim, K. Kim, Y. H. Jo et al., "Effect of therapeutic hypothermia according to severity of sepsis in a septic rat model," Cytokine, vol. 60, no. 3, pp. 755-761, 2012.

[24] J. L. D. Souza Neto, I. Araújo Filho, A. C. M. Do Rego et al., "Effects of simvastatin in abdominal sepsis in rats," Acta Cirurgica Brasileira, vol. 21, supplement 4, pp. 8-12, 2006.

[25] J. Carlet, J. Cohen, T. Calandra, S. M. Opal, and H. Masur, "Sepsis: time to reconsider the concept," Critical Care Medicine, vol. 36, no. 3, pp. 964-966, 2008.

[26] C. C. Finnerty, D. N. Herndon, D. L. Chinkes, and M. G. Jeschke, "Serum cytokine differences in severely burned children with and without sepsis," Shock, vol. 27, no. 1, pp. 4-9, 2007.

[27] Z. Y. Peng, M. J. Carter, and J. A. Kellum, "Effects of hemoadsorption on cytokine removal and short-term survival in septic rats," Critical Care Medicine, vol. 36, no. 5, pp. 1573-1577, 2008.

[28] B. Echtenacher, W. Falk, D. N. Mannel, and P. H. Krammer, "Requirement of endogenous tumor necrosis factor/cachectin for recovery from experimental peritonitis," Journal of Immunology, vol. 145, no. 11, pp. 3762-3766, 1990.

[29] M. K. Eskandari, G. Bolgos, C. Miller, D. T. Nguyen, L. E. DeForge, and D. G. Remick, "Anti-tumor necrosis factor antibody therapy fails to prevent lethality after cecal ligation and puncture or endotoxemia," Journal of Immunology, vol. 148, no. 9, pp. 2724-2730, 1992.
[30] C. S. Deutschman, M. Cereda, E. A. Ochroch, and N. R. Raj, "Sepsis-induced cholestasis, steatosis, hepatocellular injury, and impaired hepatocellular regeneration are enhanced in interleukin-6 -/- mice," Critical Care Medicine, vol. 34, no. 10, pp. 2613-2620, 2006.

[31] A. Oberholzer, C. Oberholzer, and L. L. Moldawer, "Interleukin10: a complex role in the pathogenesis of sepsis syndromes and its potential as an anti-inflammatory drug," Critical Care Medicine, vol. 30, supplement 1, pp. S58-S63, 2002.

[32] D. G. Remick, G. R. Bolgos, J. Siddiqui, J. Shin, and J. A. Nemzek, "Six at six: interleukin- 6 measured $6 \mathrm{~h}$ after the initiation of sepsis predicts mortality over 3 days," Shock, vol. 17, no. 6, pp. 463-467, 2002. 


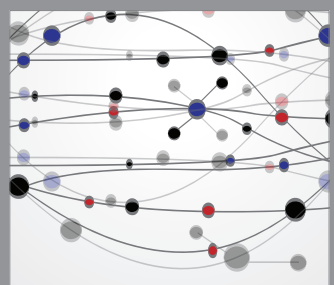

The Scientific World Journal
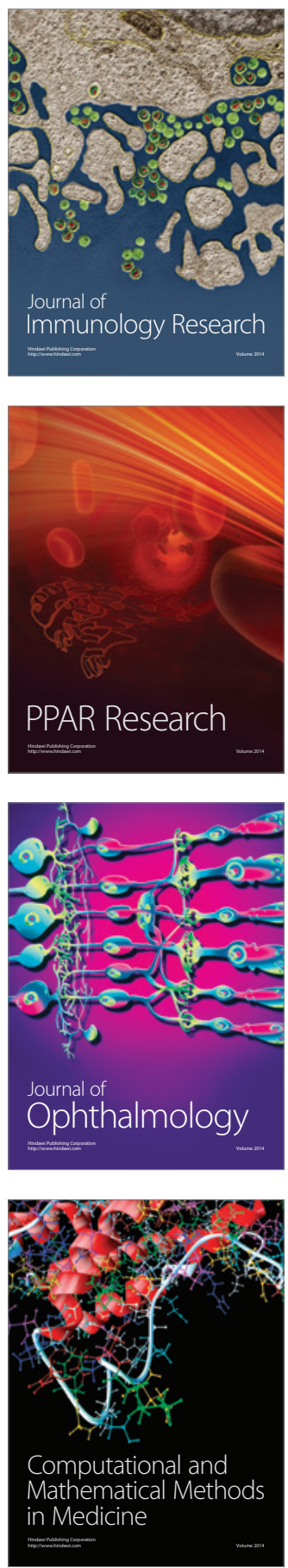

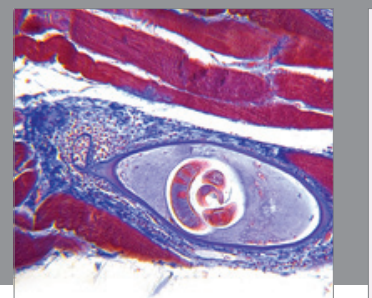

Gastroenterology

Research and Practice
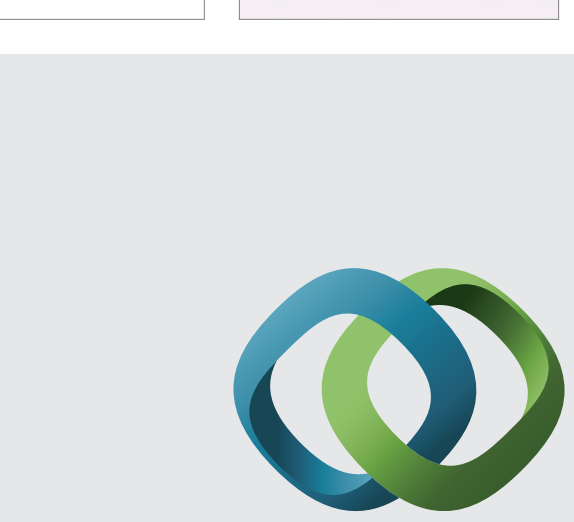

\section{Hindawi}

Submit your manuscripts at

http://www.hindawi.com
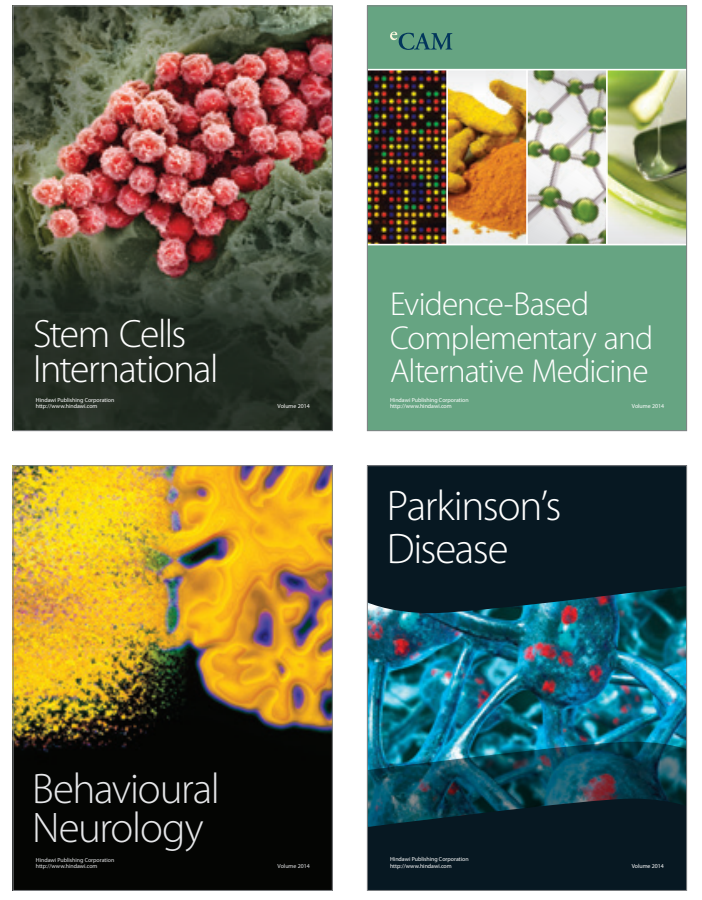
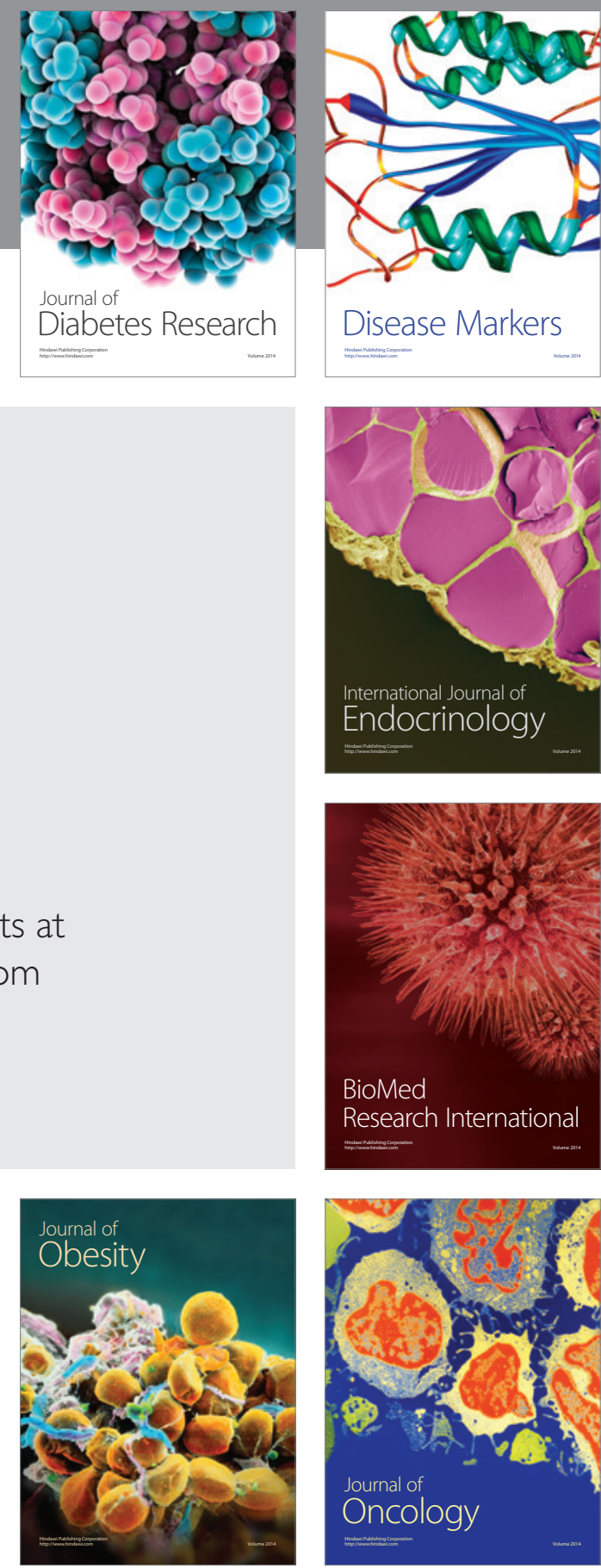

Disease Markers
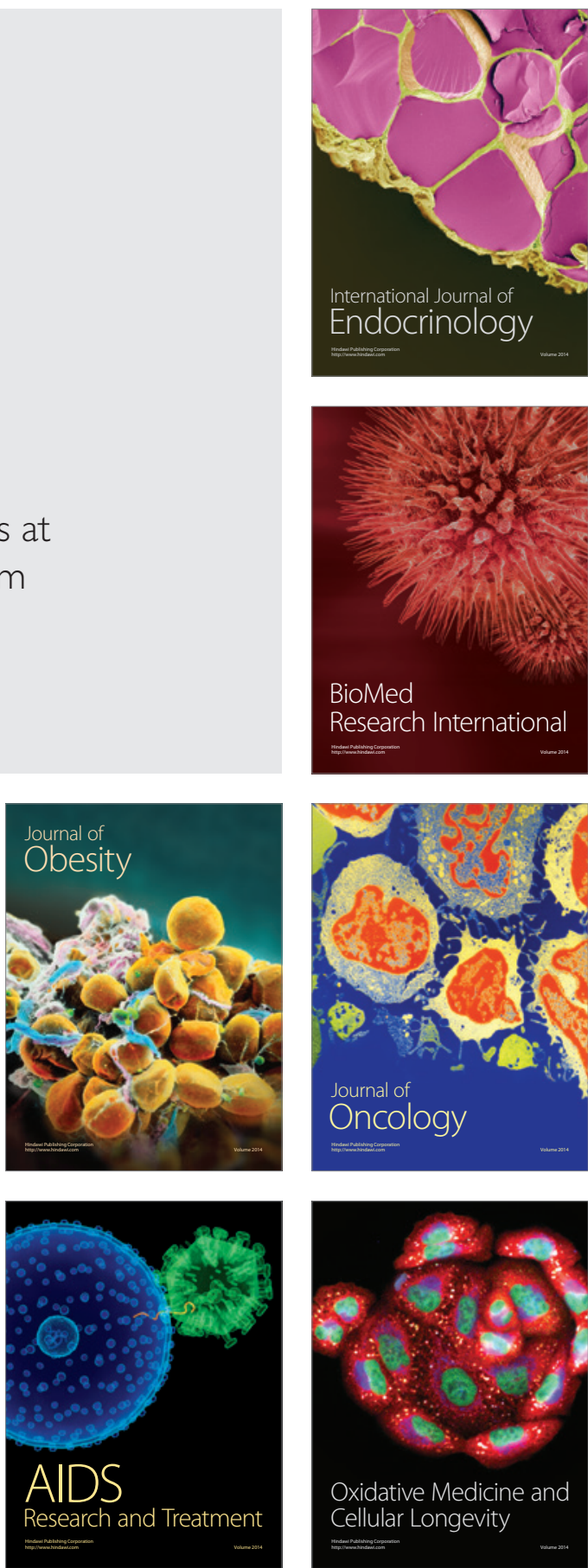